\title{
A New Approach Using Manganese-Enhanced MRI to Diagnose Acute Mesenteric Ischemia in a Rabbit Model: Initial Experience
}

\author{
Da-wei Zhao, Cheng Cheng, Lian-qin Kuang, Yu-long Zhang, Hai-yun Cheng, \\ Jia-yan Min, and Yi Wang \\ Department of Radiology, Institute of Surgery Research, Daping Hospital, Third Military Medical University, Chongqing 400042, China \\ Correspondence should be addressed to Yi Wang; ywhxl@qq.com
}

Received 13 May 2015; Accepted 28 September 2015

Academic Editor: Giuseppe Rubini

Copyright (c) 2015 Da-wei Zhao et al. This is an open access article distributed under the Creative Commons Attribution License, which permits unrestricted use, distribution, and reproduction in any medium, provided the original work is properly cited.

\begin{abstract}
Purpose. Manganese-enhanced MRI (MEMRI) has been applied to a wide range of biological and disease research. The purpose of the study was to use MEMRI to diagnose the acute mesenteric ischemia (AMI). Methods. The institutional experimental animal ethics committee approved this study. To optimize the dose of $\mathrm{Mn}^{2+}$ infusion, a dose-dependent curve was obtained using $\mathrm{Mn}^{2+}$ enhanced $T_{1}$ map MRI by an intravenous infusion $2.5-20 \mathrm{nmol} / \mathrm{g}$ body weight (BW) of $50 \mathrm{nmol} / \mathrm{L} \mathrm{MnCl}_{2}$. The eighteen animals were divided into control, sham-operated, and AMI groups. AMI models were performed by ligating the superior mesenteric artery (SMA). $T_{1}$ values were measured on $T_{1}$ maps in regions of the small intestinal wall and relaxation rate $\left(\Delta R_{1}\right)$ was calculated. Results. A nonlinear relationship between infused $\mathrm{MnCl}_{2}$ solution dose and increase in small intestinal wall $\Delta R_{1}$ was observed. Control animal exhibited significant $\mathrm{Mn}^{2+}$ clearance over time at the dose of $15 \mathrm{nmol} / \mathrm{g}$ BW. In the AMI model, $\Delta R_{1}$ values $(0.95 \pm 0.13)$ in the small intestinal wall were significantly lower than in control group $(2.05 \pm 0.19)$ after $\mathrm{Mn}^{2+}$ infusion $(P<0.01)$. Conclusion. The data suggest that MEMRI shows potential as a diagnostic technique that is directly sensitive to the poor or absent perfusion in AMI.
\end{abstract}

\section{Introduction}

Acute mesenteric ischemia (AMI) is thought to be a lifethreatening abdominal emergency characterized by the sudden occlusion of mesenteric arteries followed by impairment of intestinal blood flow. Currently, AMI accounts for approximately $0.1 \%$ of all hospital admissions, and it constitutes $1 \%$ to $2 \%$ of all gastrointestinal diseases [1]. The incidence has recently increased with the increasing age of the population [2].

The mortality rate of AMI is approximately $71 \%$, and it has remained at this high level for decades [3]. The reasons for this are illustrated as follows. On one hand, early symptoms are nonspecific in differential diagnosis of abdominal pain; on the other hand, there is an unacceptable time delay before treatment even when a diagnosis of AMI is considered. Even a warm ischemia time of 6 hours leads to morphological alterations due to disintegration of the intestinal mucosal barriers, following the bacterial translocation and gangrene of the intestinal wall [2]. This eventually results in severe peritonitis, ileus, sepsis, and multiorgan failure. A 24-hour delay decreases survival rates by $20 \%$ [1]. Therefore, early diagnosis and development of new diagnostic strategy, before the development of intestinal infarction and peritonitis, are essential for intestinal viability and patient survival.

Currently, the clinical diagnosis for AMI is based on the vascular imaging to display the occlusive site of vessels through computer tomography angiography (CTA) and magnetic resonance angiography (MRA). Still, multidetector computer tomography (MDCT) has been currently the first-line and standard diagnostic method $[4,5]$. MDCT demonstrates not only vascular structures but also intestinal wall changes and free abdominal air that may produce by hollow organ perforation [6]. This is substantial benefit for differential diagnosis between mesenteric ischemia and the other acute abdomen. The dynamic MRA yielded sensitivity and specificity of $95 \%$ and $100 \%$, respectively [7]. In the study using an in vivo rat mesenteric ischemia model, 7-T MRI allows for the identification of pathological findings of ischemic colitis and histopathological correlation [8]. 
The high $T_{1}$ relaxivity of $\mathrm{Mn}^{2+}$ and the ability of $\mathrm{Mn}^{2+}$ to enter cells through $\mathrm{Ca}^{2+}$ channels have led to the development of manganese-enhanced MRI (MEMRI) for a wide range of biological study, including brain anatomy and function [9-12], myocardial infarction $[13,14]$, and brain ischemia [15]. Therefore, in the present study, a strategy using MEMRI to diagnose AMI can be based on the fact that $\mathrm{Mn}^{2+}$ cannot arrive at suffered small intestine so that the relaxivity of small intestine will not be changed due to the reduction in contrast agents delivery caused by SMA in the AMI. Conversely, in the normal small intestinal wall without occlusion of SMA, due to the accumulation of $\mathrm{Mn}^{2+}$, the small intestine will have a shorter relaxivity, which in turn will increase signal intensity on $T_{1}$-weighted image. We propose that MEMRI provide the direct image with the ability to distinguish between normal and ischemic small intestines for AMI.

\section{Materials and Methods}

2.1. Animal Subject and Preparation. The study was approved by the experimental animals ethics committee at Daping Hospital of Third Military Medical University. MEMRI experiments were performed in adult New Zealand white rabbits (mean \pm SEM weight: $3.17 \pm 0.12 \mathrm{~kg}$ ), which were provided by the Animal Center of Daping Hospital. Rabbits were fasted for 12 hrs before experiment but were allowed free access to water. The animals were initially anesthetized with an intravenous injection of $3 \%$ pentobarbital sodium at the dose of $30 \mathrm{mg} / \mathrm{kg}$. The anesthetized animals were maintained in side position on the warming pad throughout the MRI session. A custom-made abdominal bandage was used to provide abdominal pressure to prevent both abdominal breathing and intestinal peristalsis in an effort to minimize artifacts due to motion. During the whole procedure, animals maintained a relatively constant physiological level, body temperature $\left(38.6 \pm 0.71^{\circ} \mathrm{C}\right)$, heart rate $(205.5 \pm 12.90$ beats $/ \mathrm{min})$, and breath frequency $(35.0 \pm 3.67$ times $/ \mathrm{min})$.

A dorsal ear vein line for $\mathrm{Mn}^{2+}$ infusion was introduced after anesthesia. A $50 \mathrm{mM} \mathrm{MnCl}_{2}$ solution was obtained by dissolving $\mathrm{MnCl}_{2}$ crystals in saline ( $\mathrm{pH}$ adjusted to 7.4). To acquire dose-dependent curve, $\mathrm{MnCl}_{2}$ was administered into control rabbits at the various doses ranging from 2.5 to $20 \mathrm{nmol} / \mathrm{g}$ total body weight (BW). For the temporal washout curve, a single dose of $20 \mathrm{nmol} / \mathrm{g}$ BW was infused into the control rabbits. All infusions were completed at a constant rate of $0.8 \mathrm{~mL} / \mathrm{min}$ with the aid of a syringe pump (Baoding Lead Fluid Technology, China).

2.2. Acute Mesenteric Ischemia Model. For the AMI model study, the rabbits were randomly placed into the three following groups, with each group containing six rabbits: in control group, animals underwent no surgical procedures; in sham-operated group, animals underwent sham laparotomy with exposure of the superior mesenteric artery (SMA) after anesthesia; and, in AMI group, laparotomy was performed and the SMA were ligated. The AMI model was produced as literature previously described [16]. After anesthesia, the abdominal regions of the rabbits were sheared and sterilized with $10 \%$ povidone iodine and draped with sterile towels.
Laparotomy was performed through a midline incision. After the small bowel and cecum were identified, the root of SMA was carefully exposed and ligated with 0 silk sutures. The abdominal contents were replaced, and then the peritoneum and abdominal wall were sutured following standard procedures. After surgery, MRI was immediately carried out according to the above-mentioned position.

For $\mathrm{MnCl}_{2}$ infusion, all three groups of rabbits were inserted into dorsal ear vein and infused with $15 \mathrm{nmol} / \mathrm{g} \mathrm{BW}$ at a constant rate of $0.8 \mathrm{~mL} / \mathrm{min}$.

2.3. MRI. The in vivo $T_{1}$ map MRI studies were carried out using anesthetized animals that had been placed in the magnet bore in the lateral position. In order to minimize artifacts due to motion, a custom-made abdominal bandage was used to achieve attenuating small bowel motion caused by both abdominal breathing and intestinal peristalsis.

All MR imaging was acquired at a $1.5 \mathrm{~T}$ MRI scanner (MAGNETOM Aera, Siemens AG, Germany) with a $70 \mathrm{~cm}$ bore magnet equipped with XI gradients (33 mT/m @ $125 \mathrm{~T} / \mathrm{m} / \mathrm{s})$ and XQ gradients (45 mT/m @ $200 \mathrm{~T} / \mathrm{m} / \mathrm{s})$. For signal excitation and reception, an abdominal phased array coil (20 mm, 63 MHz, Siemens AG, Germany) was used. A scout image was initially obtained in order to verify the proper position. Both pre- $\mathrm{Mn}^{2+}$ and post- $\mathrm{Mn}^{2+} T_{1}$ maps were acquired using $T_{1}$ map sequence. The imaging parameters were as follows: $\mathrm{TR} / \mathrm{TE}=15 / 1.59 \mathrm{~ms}$, slices per slab $=$ 52 , slice thickness $=3.0 \mathrm{~mm}$, flip angle $=5^{\circ}$ and $26^{\circ}$, matrix $=256 \times 256$, and $\mathrm{FOV}=231 \mathrm{~mm} \times 231 \mathrm{~mm} . \mathrm{MnCl}_{2}$ was injected using a 22-gauge syringe through vein at a constant rate of $0.8 \mathrm{~mL} / \mathrm{min}$. After contrast agent injection, $T_{1}$ map images were obtained approximately $5 \mathrm{~min}$ after completion of the infusion to allow $\mathrm{Mn}^{2+}$ blood pool clearance. The total imaging time per $T_{1}$ map was $4 \mathrm{~min}$ and $50 \mathrm{~s}$. $T_{1}$ values were acquired before and after $\mathrm{Mn}^{2+}$ infusion to calculate the change of $T_{1}$ relaxation rate due to $\mathrm{MnCl}_{2}$ infusion.

Both pre- $\mathrm{Mn}^{2+}$ and post- $\mathrm{Mn}^{2+} \mathrm{T}_{1}$-weighted images were acquired using FLASH $T_{1}$ dual echo sequence. The imaging parameters were as follows: $\mathrm{TR}=250 \mathrm{~ms}, \mathrm{TE}_{1}=2.37 \mathrm{~ms}$, $\mathrm{TE}_{2}=4.87 \mathrm{~ms}$, slices per slab $=52$, slice thickness $=3.0 \mathrm{~mm}$, number of averages $=1$, matrix $=256 \times 256$, and FOV $=$ $231 \mathrm{~mm} \times 231 \mathrm{~mm}$.

$T_{1}$ map MRI datasets were processed offline for image reconstructions using workstation. ROI before and after $\mathrm{Mn}^{2+}$ infusion were defined in MRI anatomical and enhanced regions of small intestinal wall, respectively. For evaluation, $T_{1}$ values of each rabbit were measured in small intestinal wall using defined ROI on the two-dimensional $T_{1}$ maps to calculate average $T_{1}$ values. The change of $T_{1}$ relaxation rate $\left(\Delta R_{1}\right)$ was calculated as post- $\mathrm{Mn}^{2+}$ infusion $R_{1}-$ pre- $\mathrm{Mn}^{2+}$ infusion $R_{1}$, where the relaxation rate $\left(R_{1}\right)$ is defined as $1 / T_{1}$.

2.4. Statistical Analysis. All data are presented as mean \pm SEM. $\Delta R_{1}$ values between the three groups in AMI study were compared by two-way ANOVA and Bonferroni post hoc test. All statistical analyses were performed using GraphPad Prism 5 (GraphPad Software Inc., San Diego, CA, USA). $P$ value less than 0.05 was considered statistically significant. 


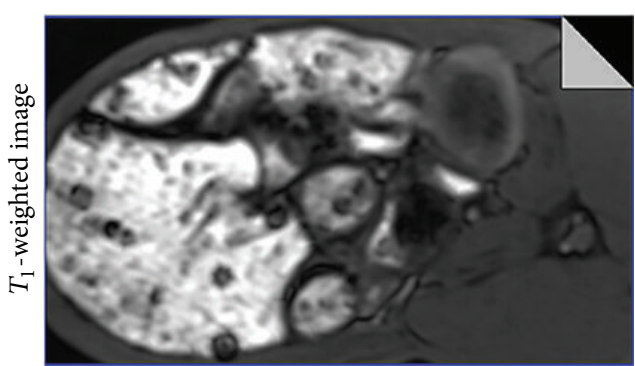

Before $\mathrm{Mn}^{2+}$ infusion

(a)

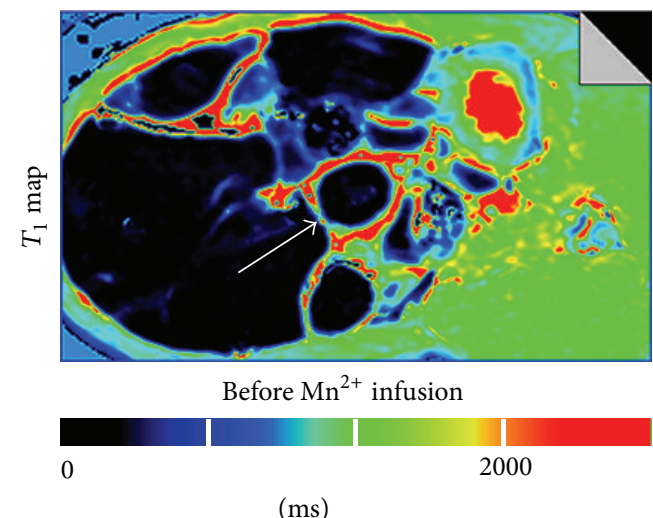

(c)

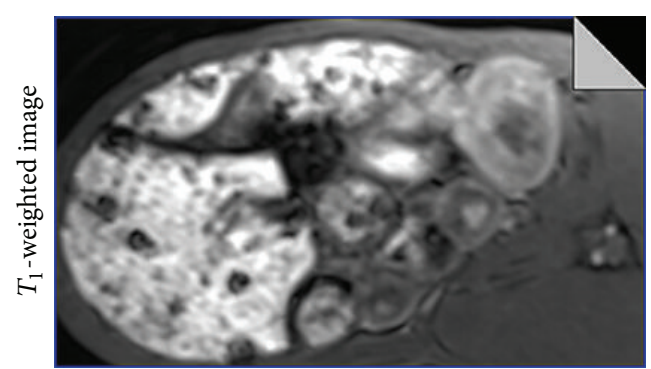

After $\mathrm{Mn}^{2+}$ infusion

(b)

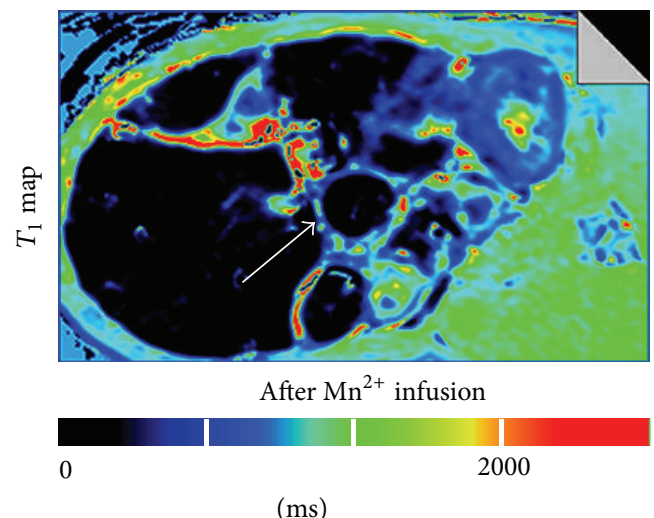

(d)

Figure 1: Example of small intestinal wall images showed the changes of $T_{1}$ signal in the small intestinal wall for a control rabbit in $T_{1}$ weighted images and $T_{1}$ maps. (a) $T_{1}$-weighted image before $\mathrm{Mn}^{2+}$ infusion. (b) $T_{1}$-weighted image after $\mathrm{Mn}^{2+}$ infusion. (c) $T_{1}$ map before $\mathrm{Mn}^{2+}$ infusion. (d) $\mathrm{T}_{1}$ map after $\mathrm{Mn}^{2+}$ infusion. The white arrow indicated signal change of the small intestinal wall before and after $\mathrm{Mn}^{2+}$ infusion.

\section{Results}

Sample small intestinal MR images for a control rabbit are shown in Figure 1. Figures 1(a) and 1(b) show $T_{1}$-weighted signal intensity enhancements before and after $\mathrm{Mn}^{2+}$ infusion, respectively. Figures $1(\mathrm{c})$ and $1(\mathrm{~d})$ show the corresponding $T_{1}$ maps before and after $\mathrm{Mn}^{2+}$ infusion, respectively. At this $\mathrm{Mn}^{2+}$ dose of $15 \mathrm{nmol} / \mathrm{g} \mathrm{BW}$, there is significant enhancement of the signal intensity in the small intestinal wall after $\mathrm{Mn}^{2+}$ infusion.

In present study, $T_{1}$ map was performed to determine the relationship between $\mathrm{Mn}^{2+}$ infusion dose and tissue $\Delta R_{1}$ for small intestinal wall. The effect of altering the infusion dose on small intestinal wall $\Delta R_{1}$ is shown in Figure 2(a). This dose-dependent curve can be used to optimize minimal $\mathrm{Mn}^{2+}$ infusion doses while still achieving adequate signal enhancement. $\Delta R_{1}$ values ranged from $0.09 / \mathrm{s}$ at dose of $2.5 \mathrm{nmol} / \mathrm{g}$ BW to an average of $1.87 / \mathrm{s}$ for dose above $15 \mathrm{nmol} / \mathrm{g}$ BW. At $\mathrm{Mn}^{2+}$ infusion dose from $5 \mathrm{nmol} / \mathrm{g} \mathrm{BW}$ to $15 \mathrm{nmol} / \mathrm{g}$ BW, the dose of $\mathrm{Mn}^{2+}$ infusion, $X(\mathrm{nmol} / \mathrm{g}$ BW) on relaxivity, $\Delta R_{1}(1 / \mathrm{s})$, yields a linear relationship of $\Delta R_{1}=0.1640 X-0.5972\left(r^{2}=0.9895\right)$. Above $15 \mathrm{nmol} / \mathrm{g}$ $\mathrm{BW}$, the relaxivity enhancement reaches a plateau $\left(\Delta R_{1}=\right.$ $1.87 \pm 0.15 / \mathrm{s})$. Within this physiological steady plane, any further rise in $\mathrm{Mn}^{2+}$ administered dose does not improve $\Delta R_{1}$.
$\mathrm{Mn}^{2+}$ temporal washout phenomenon was also studied in control rabbits infused with a single $\mathrm{MnCl}_{2}$ dose of $15 \mathrm{nmol} / \mathrm{g}$ BW. The data presented in Figure 2(b) show the temporal relationship between $\Delta R_{1}$ and the washout time. The washout period was examined from 0 to $75 \mathrm{~min}$. The small intestinal wall $\Delta R_{1}$ signal has attenuated by $50 \%$ in the first $35 \mathrm{~min}$ after infusion. During the first $45 \mathrm{~min}$ after infusion, the washout data for this infusion dose can be fitted in a linear relationship with small intestinal wall $\Delta R_{1 \text { washout }}=-0.02402 X+$ $1.864\left(r^{2}=0.9640\right)$. This curve provides a better imaging time frame for the small intestinal disease model in this study.

The final aim of this study was to explore variety of relaxivity due to acute mesenteric ischemia. All three groups of rabbits, namely, control group, sham-operated group, and AMI model, were infused with a single $\mathrm{MnCl}_{2}$ dose of $15 \mathrm{nmol} / \mathrm{g}$ BW. Each group contained six animals. $T_{1}$ mapping was immediately performed after initial $\mathrm{Mn}^{2+}$ infusion. Figure 3 showed changes of signal on $T_{1}$-weighted image and $T_{1}$ maps for sham-operated group (Figures $3(\mathrm{a})$ and $3(\mathrm{c})$ ) and AMI model (Figures 3(b) and 3(d)) after $\mathrm{Mn}^{2+}$ infusion. By comparing AMI model to the sham-operated group, there was a significant difference of signal enhancement. In the AMI model, there were higher $T_{1}$ values on $T_{1}$ map due to reduced uptake of $\mathrm{Mn}^{2+}$. On the contrary, in the shamoperated rabbits, there were lower $T_{1}$ values on $T_{1}$ map, 


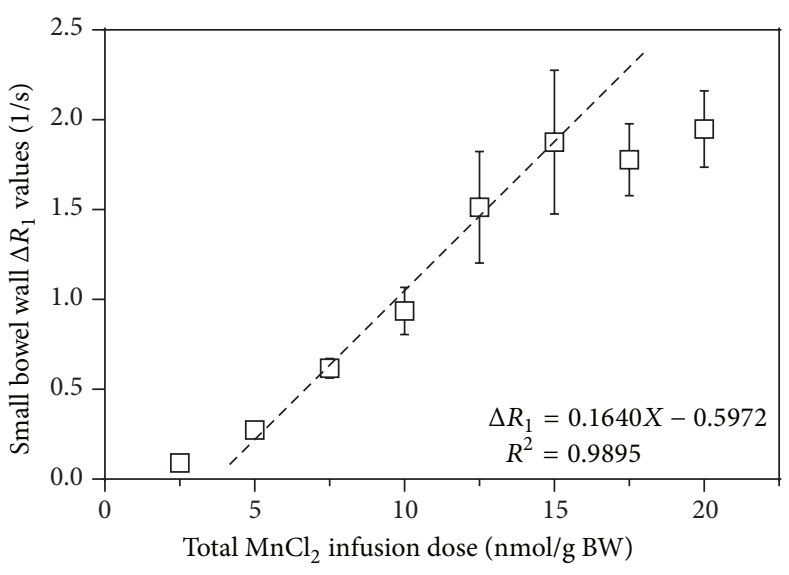

(a)

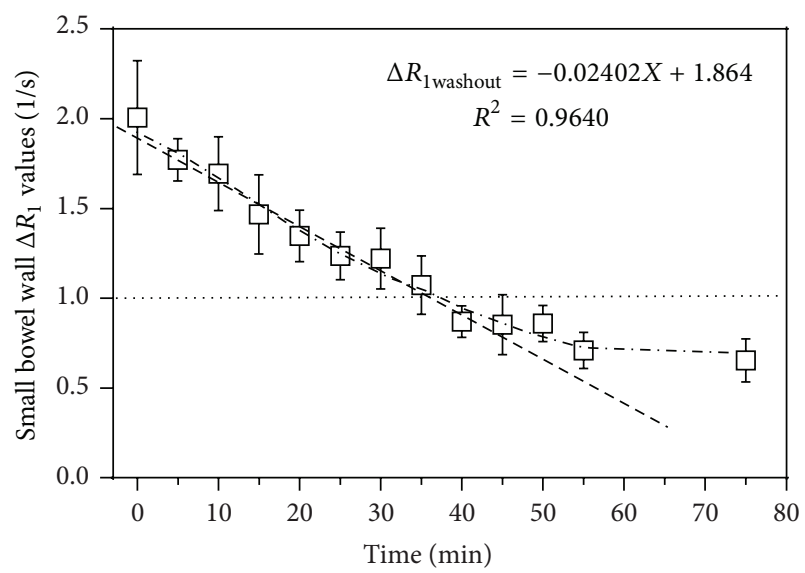

(b)

Figure 2: Effect of altering the dose of infused $\mathrm{Mn}^{2+}$ on small intestinal wall relaxivity. (a) The dose-dependent curve for different dose of $\mathrm{MnCl}_{2}$ administration. $x$-axis showed the total dose of infused $\mathrm{Mn}^{2+}$ normalized to rabbit BW. $y$-axis showed the change of relaxation rate, $\Delta R_{1}$. As a function of dose, a linear dose-uptake region was noted along with a plateau region above $15 \mathrm{nmol} / \mathrm{g}$ BW. The linear fit was shown with total $\mathrm{Mn}^{2+}$ infusion dose, $X$, in nmol/g BW. Data are presented as the mean \pm SEM of $n=3$. (b) Temporal Mn ${ }^{2+}$ washout curve. $x$-axis showed the experimental time course after $\mathrm{Mn}^{2+}$ infusion. $y$-axis showed the difference in the change of relaxation rate, $\Delta R_{1}$.

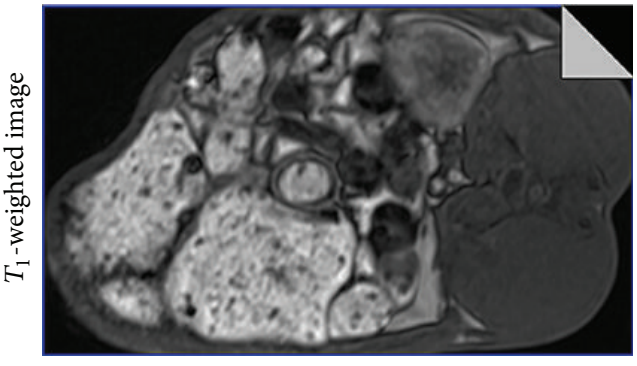

Sham-operated

(a)

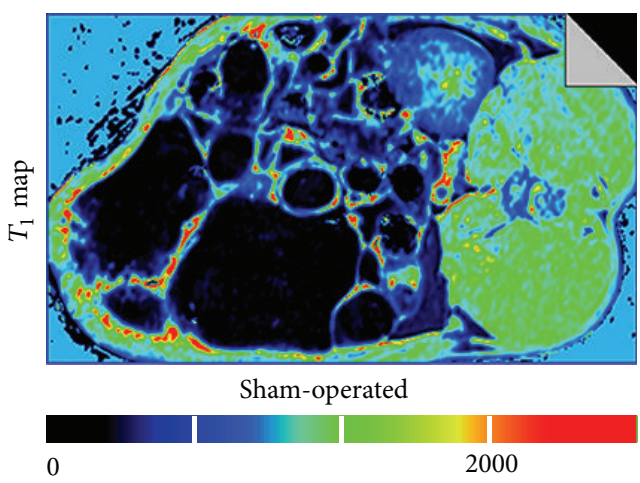

(ms)

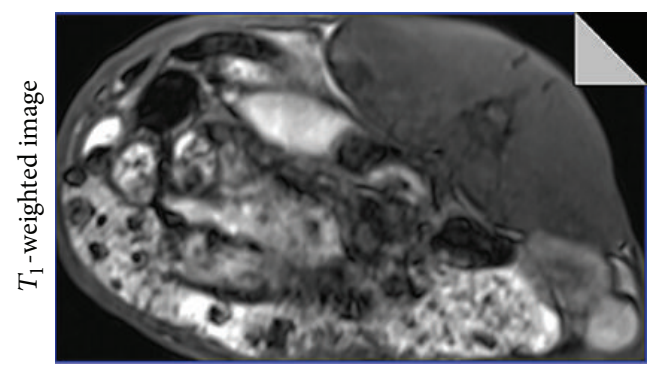

AMI model

(b)

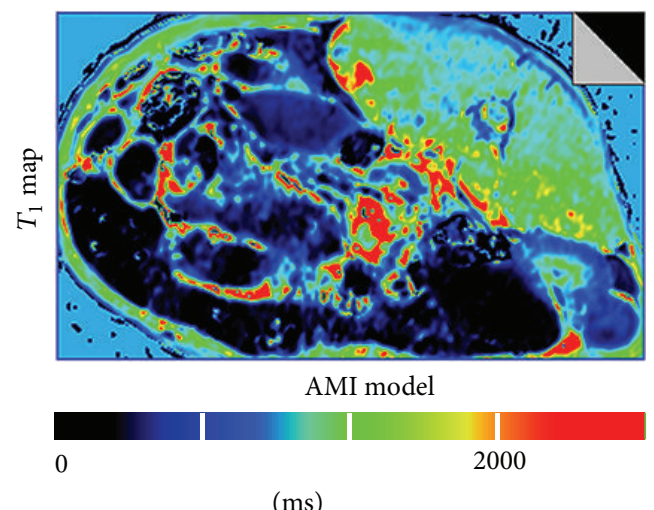

(d)

Figure 3: Example of post- $\mathrm{Mn}^{2+}$ infusion $T_{1}$-weighted image and $T_{1}$ map for a sham-operated rabbit (a and c) and AMI model (b and d). The small intestinal wall of AMI model showed a longer $T_{1}$ relaxation time than that of sham-operated animal. Significant decreasing of $T_{1}$ values can be seen in the small intestinal wall of sham-operated group compared with AMI groups.

accompanied by higher signal enhancement on $T_{1}$-weighted image due to uptake of $\mathrm{Mn}^{2+}$.

The results of this study were shown in Figure 4 and Table 1. There was no statistically significant difference in $\Delta R_{1}$ values between the control group $(2.05 \pm 0.19)$ and shamoperated group $(1.75 \pm 0.20)$, suggesting that the surgical operation of opening the abdomen does not affect $\mathrm{Mn}^{2+}$ uptake. Comparing AMI group with the control group, 
TABLE 1: Mean $R_{1}$ values before and after $\mathrm{Mn}^{2+}$ infusion and mean $\Delta R_{1}$ for control group, sham-operated group, and AMI model (1/s).

\begin{tabular}{lccc}
\hline \multirow{2}{*}{ Group } & \multicolumn{2}{c}{$R_{1}(1 / \mathrm{s})$} & \multirow{2}{*}{$\Delta R_{1}(1 / \mathrm{s})^{\mathrm{a}}$} \\
\hline Control & $0.49 \pm 0.033$ & $2.54 \pm 0.22$ & $2.05 \pm 0.19(n=6)$ \\
Sham-operated & $0.48 \pm 0.028$ & $2.23 \pm 0.19$ & $1.75 \pm 0.20(n=6)$ \\
AMI & $0.49 \pm 0.037$ & $1.44 \pm 0.16^{\mathrm{b}, \mathrm{c}}$ & $0.95 \pm 0.13(n=6)^{\mathrm{b}, \mathrm{c}}$ \\
\hline
\end{tabular}

${ }^{\mathrm{a}}$ Values are expressed as mean \pm SEM.

${ }^{\mathrm{b}} P<0.01$ among all groups (analysis of one-way ANOVA).

${ }^{\mathrm{c}} P<0.01$ compared with control group (unpaired two-tailed $t$-test).

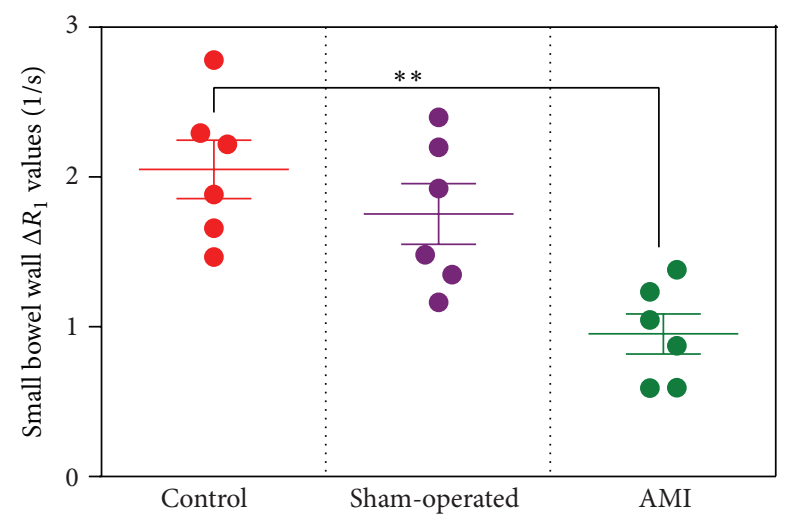

FIGURE 4: Effect of AMI on the uptake of $\mathrm{Mn}^{2+}$. Data are presented as the mean $\Delta R_{1} \pm$ SEM of $n=6 .{ }^{* *} P<0.01$ versus control group.

however, revealed that there was a significant difference in $\Delta R_{1}$ values after $\mathrm{Mn}^{2+}$ infusion. $\Delta R_{1}$ values of AMI groups $(0.95 \pm 0.13)$ were distinctly lower than those of control group $(2.05 \pm 0.19)$. These data demonstrate the sensitivity of this technique to determine altered $\mathrm{Mn}^{2+}$ uptake at the AMI model. Detecting changes of relaxivity in AMI injury may allow us to timely identify acute AMI intestinal wall in preclinical models for future treatment or prevention.

\section{Discussion}

AMI are vascular emergencies that require an immediate diagnosis and medical and surgical intervention. The preliminary data from this study reveal that normal and ischemic intestines can be identified using MEMRI technique in the rabbit AMI model. Additionally, the dosage of $\mathrm{Mn}^{2+}$ infusion for optimal signal enhancement in the small intestinal wall is first established via showing the dynamic range of relaxivity changes over a range of $\mathrm{Mn}^{2+}$ infusion doses. The dosedependent curve reveals the nonlinear relationship between total $\mathrm{Mn}^{2+}$ infusion dose and $\Delta R_{1}$ of the small intestinal wall (Figure 2(a)). At a dose of above $15 \mathrm{nmol} / \mathrm{g} \mathrm{BW}$, a plateau of $\Delta R_{1}$ occurs, where $\Delta R_{1}$ does not increase after an increase in dose of $\mathrm{Mn}^{2+}$ infusion. This phenomenon is similar to the performance of $\mathrm{Mn}^{2+}$ in the heart reported by previous literature [14]. However, the dosages of $\mathrm{Mn}^{2+}$ reaching the plateau in the small intestinal wall are far less than those in the heart. Furthermore, the signal washout curve shows that the small intestinal wall $\Delta R_{1}$ signal has attenuated by $50 \%$ after 35 min (Figure 2(b)).

The known cytotoxic effects of high doses of $\mathrm{Mn}^{2+}$ initially discouraged its use as an MRI contrast agent [17]. Thus, minimizing the administered dose of $\mathrm{Mn}^{2+}$ is critical for MEMRI into clinical application. Here we have shown that a dose of $15 \mathrm{nmol} / \mathrm{g} \mathrm{BW}$, which is far less than $197 \mathrm{nmol} / \mathrm{g} \mathrm{BW}$ described in other reports [14], can be selected to produce significant relaxivity changes in the small intestinal wall. Additionally, any further increase in the dose of $\mathrm{Mn}^{2+}$ above $15 \mathrm{nmol} / \mathrm{g}$ BW does not result in a further decrease in $T_{1}$ values. Overall, these data suggest that $\mathrm{Mn}^{2+}$ is safe to use as a contrast agent in intestinal MEMRI.

$\mathrm{Mn}^{2+}$ can influence intestinal motility via affecting $\mathrm{Ca}^{2+}$ influx of the smooth muscles of the intestinal mucosa. $\mathrm{Mn}^{2+}$ affects $\mathrm{Ca}^{2+}$ influx in a concentration-dependent manner. $\mathrm{Mn}^{2+}$ inhibited smooth muscle contraction by blocking $\mathrm{Ca}^{2+}$ influx [18]; the inhibition could be overcome by high extracellular $\mathrm{Ca}^{2+}$ concentrations at millimolar concentrations [19]. $\mathrm{Mn}^{2+}$ can cause a transient contractile response through the release of acetylcholine [20] or directly induce contractions via voltage-operated $\mathrm{L}$-type $\mathrm{Ca}^{2+}$ channels at millimolar concentrations [21-23]. In present study, the $\mathrm{Mn}^{2+}$ concentrations are at nanomolar range and are far lower than the concentrations described above.

The AMI study which used the optimal dose shows statistically significant differences to discern normal and ischemic small intestines using MEMRI between sham-operated and AMI groups. MEMRI had previously been shown to provide functional and pathological information in myocardial tissue $[13,14,24]$ and brain $[12,25]$. AMI caused by the occlusion of SMA show a no-flow status in the suffering small intestine. These changes can potentially be observed and monitored using manganese-enhanced $T_{1}$ mapping as shown by the sensitivity of our data (Figure 3). The small intestinal wall of AMI model had lower $\Delta R_{1}$ than that of the sham-operated group (Figure 4).

Currently, the most accurate method for diagnosing AMI is based on mesenteric angiography. The gold standard for the diagnosis of AMI has been conventional catheter angiography that has higher sensitivity and accuracy [3]. However, it is an invasive, time-consuming, and technically complex procedure. Moreover, this diagnostic technique is unavailable at most hospitals leading to a critical delay. MDCT and CTA are advantageous over conventional angiography in that they not only delineate vascular structures but also show bowel wall changes and may exclude other causes of acute abdomen. Today, because it is less invasive and time-consuming, CTA has replaced conventional angiography as the gold standard in diagnosing AMI with sensitivity and specificity of $96 \%$ and $94 \%$, respectively $[1,4,26]$. The magnetic resonance angiography (MRA) is a developing technique. Because of being noninvasive, the lack of radiation, and allergic risk related to iodinated contrast agents, MRA is the second choice for children and patients with azotemia [27]. There is a report that shows that MRA yielded sensitivity and specificity of $95 \%$ and $100 \%$, respectively, for diagnosis of severe stenosis or occlusion of the origins of the celiac axes and superior 
mesenteric artery (SMA) [7]. However, this technique is limited to identification of more distally located occlusions. Based on current evidence, blood marker and peritoneal fluid analysis of laboratory findings are yet delayed and inaccurate to be an early diagnostic aid [28].

The literature reported that AMI is caused by an arterial embolus or thrombosis within the SMA in $60 \%$ to $70 \%$ of cases, nonocclusive ischemia in $20 \%$ to $30 \%$ of cases, and mesenteric vein thrombosis in $5 \%$ to $10 \%$ of cases [29]. There are some inevitable limits because of the fact that this technique is based on the perfusion of blood flow. Our present method is only applicable to that situation due to the occlusion of mesenteric artery. While $\mathrm{Mn}^{2+}$ flows to suffering small intestine along blood flow in the situation of the occlusion of mesenteric vein, this technique cannot discern normal and ischemic small intestines. Furthermore, this technique is also inappropriate for nonocclusive ischemia.

\section{Conclusions}

In conclusion, our investigation is the first to demonstrate that MEMRI at a low dose of $\mathrm{Mn}^{2+}$ reveals the differences of relaxivity between normal and ischemic small intestines associated with the occlusion of SMA. We hope that this exploratory research will provide additional information on preclinical and transnational models to promote diagnostic development of AMI.

\section{Conflict of Interests}

The authors declare that there is no conflict of interests regarding the publication of this paper.

\section{Authors' Contribution}

Da-wei Zhao and Cheng Cheng contributed equally to this work.

\section{Acknowledgments}

This research was financially supported by the National Natural Science Foundation of China (Grant no. 81071145). The authors would like to thank Hai-yun Cheng and Yu-long Zhang for help with all aspects of the MRI experiments.

\section{References}

[1] T. C. van den Heijkant, B. A. C. Aerts, J. A. Teijink, W. A. Buurman, and M. D. P. Luyer, "Challenges in diagnosing mesenteric ischemia," World Journal of Gastroenterology, vol. 19, no. 9, pp. 1338-1341, 2013.

[2] E. Klar, P. B. Rahmanian, A. Bücker, K. Hauenstein, K.-W. Jauch, and B. Luther, "Acute mesenteric ischemia: a vascular emergency," Deutsches Ärzteblatt International, vol. 109, no. 14, pp. 249-256, 2012.

[3] L. J. Brandt and S. J. Boley, "AGA technical review on intestinal ischemia," Gastroenterology, vol. 118, no. 5, pp. 954-968, 2000.

[4] M. C. Wyers, "Acute mesenteric ischemia: diagnostic approach and surgical treatment," Seminars in Vascular Surgery, vol. 23, no. 1, pp. 9-20, 2010.
[5] J. Menke, "Diagnostic accuracy of multidetector CT in acute mesenteric ischemia: systematic review and meta-analysis," Radiology, vol. 256, no. 1, pp. 93-101, 2010.

[6] A. Yikilmaz, O. I. Karahan, S. Senol, I. S. Tuna, and H. Y. Akyildiz, "Value of multislice computed tomography in the diagnosis of acute mesenteric ischemia," European Journal of Radiology, vol. 80, no. 2, pp. 297-302, 2011.

[7] J. F. M. Meaney, M. R. Prince, T. T. Nostrant, and J. C. Stanley, "Gadolinium-enhanced MR angiography of visceral arteries in patients with suspected chronic mesenteric ischemia," Journal of Magnetic Resonance Imaging, vol. 7, no. 1, pp. 171-176, 1997.

[8] F. Iacobellis, D. Berritto, F. Somma et al., "Magnetic resonance imaging: a new tool for diagnosis of acute ischemic colitis?" World Journal of Gastroenterology, vol. 18, no. 13, pp. 1496-1501, 2012.

[9] Y.-J. Lin and A. P. Koretsky, "Manganese ion enhances T1weighted MRI during brain activation: an approach to direct imaging of brain function," Magnetic Resonance in Medicine, vol. 38, no. 3, pp. 378-388, 1997.

[10] O. Natt, T. Watanabe, S. Boretius, J. Radulovic, J. Frahm, and T. Michaelis, "High-resolution 3D MRI of mouse brain reveals small cerebral structures in vivo," Journal of Neuroscience Methods, vol. 120, no. 2, pp. 203-209, 2002.

[11] T. Watanabe, O. Natt, S. Boretius, J. Frahm, and T. Michaelis, "In vivo 3D MRI staining of mouse brain after subcutaneous application of $\mathrm{MnCl}_{2}$," Magnetic Resonance in Medicine, vol. 48, no. 5, pp. 852-859, 2002.

[12] I. Aoki, Y.-J. L. Wu, A. C. Silva, R. M. Lynch, and A. P. Koretsky, "In vivo detection of neuroarchitecture in the rodent brain using manganese-enhanced MRI," NeuroImage, vol. 22, no. 3, pp. 1046-1059, 2004.

[13] G. A. Krombach, M. Saeed, C. B. Higgins, V. Novikov, and M. F. Wendland, "Contrast-enhanced MR delineation of stunned myocardium with administration of $\mathrm{MnCl}_{2}$ in rats," Radiology, vol. 230, no. 1, pp. 183-190, 2004.

[14] B. Waghorn, T. Edwards, Y. Yang, K.-H. Chuang, N. Yanasak, and T. C.-C. Hu, "Monitoring dynamic alterations in calcium homeostasis by T1-weighted and T1-mapping cardiac manganese-enhanced MRI in a murine myocardial infarction model," NMR in Biomedicine, vol. 21, no. 10, pp. 1102-1111, 2008.

[15] I. Aoki, S. Naruse, and C. Tanaka, "Manganese-enhanced magnetic resonance imaging (MEMRI) of brain activity and applications to early detection of brain ischemia," NMR in Biomedicine, vol. 17, no. 8, pp. 569-580, 2004.

[16] Z. D. Dundar, B. Cander, M. Gul, K. U. Karabulut, and S. Girisgin, "Serum ischemia-modified albumin levels in an experimental acute mesenteric ischemia model," Academic Emergency Medicine, vol. 17, no. 11, pp. 1233-1238, 2010.

[17] A. C. Silva, J. H. Lee, I. Aoki, and A. P. Koretsky, "Manganeseenhanced magnetic resonance imaging (MEMRI): methodological and practical considerations," NMR in Biomedicine, vol. 17, no. 8, pp. 532-543, 2004.

[18] T. Itoh, H. Kuriyama, and T. Nanjo, "Effects of calcium and manganese ions on mechanical properties of intact and skinned muscles from the guinea-pig stomach," The Journal of Physiology, vol. 333, no. 1, pp. 555-576, 1982.

[19] K. E. Killoran, A. D. Miller, K. S. Uray et al., "Role of innate immunity and altered intestinal motility in LPS- and $\mathrm{MnCl} 2$ induced intestinal intussusception in mice," American Journal of Physiology-Gastrointestinal and Liver Physiology, vol. 306, no. 5, pp. G445-G453, 2014. 
[20] T. Nasu, "Actions of manganese ions in contraction of smooth muscle," General Pharmacology: The Vascular System, vol. 26, no. 5, pp. 945-953, 1995.

[21] T. Nasu, H. Murase, and H. Shibata, "Manganese ions penetrate via L-type $\mathrm{Ca}^{2+}$ channels and induce contraction in high- $\mathrm{K}^{+}$ medium in ileal longitudinal muscle of guinea-pig," General Pharmacology, vol. 26, no. 2, pp. 381-386, 1995.

[22] T. Nasu and K. Baba, "Mechanics of manganese ions-induced contraction of ileal longitudinal muscle in $\mathrm{Ca}^{2+}$-free, high- $\mathrm{K}^{+}$ medium," General Pharmacology: The Vascular System, vol. 28, no. 4, pp. 503-507, 1997.

[23] T. Nasu and A. Shibata, "Action of ouabain on the contraction of ileal longitudinal muscle by manganese ions in a $\mathrm{Ca}^{2+}$-free, high- $\mathrm{K}^{+}, \mathrm{Na}^{+}$-sufficient or $\mathrm{Na}^{+}$-deficient solution," Pharmacological Research, vol. 42, no. 6, pp. 511-515, 2000.

[24] B. Waghorn, A. Schumacher, J. Liu et al., "Indirectly probing $\mathrm{Ca}^{2+}$ handling alterations following myocardial infarction in a murine model using $\mathrm{T}_{1}$-mapping manganese-enhanced magnetic resonance imaging," Magnetic Resonance in Medicine, vol. 65, no. 1, pp. 239-249, 2011.

[25] K. Fang, Y. Li, H. Liu, W. Liao, and H. Lei, “Tracing neuronal tracts in the olfactory pathway of rat and detecting ischemic core in a rat model of focal ischemia using manganese enhanced magnetic resonance imaging," Chinese Science Bulletin, vol. 49, no. 17, pp. 1834-1840, 2004.

[26] I. D. C. Kirkpatrick, M. A. Kroeker, and H. M. Greenberg, "Biphasic CT with mesenteric CT angiography in the evaluation of acute mesenteric ischemia: initial experience," Radiology, vol. 229, no. 1, pp. 91-98, 2003.

[27] M.-C. P. Shih and K. D. Hagspiel, "CTA and MRA in mesenteric ischemia: part I, role in diagnostic and differential diagnosis," American Journal of Roentgenology, vol. 188, no. 2, pp. 452-461, 2007.

[28] S. Acosta and T. Nilsson, "Current status on plasma biomarkers for acute mesenteric ischemia," Journal of Thrombosis and Thrombolysis, vol. 33, no. 4, pp. 355-361, 2012.

[29] W. Wiesner, A. Hauser, and W. Steinbrich, "Accuracy of multidetector row computed tomography for the diagnosis of acute bowel ischemia in a non-selected study population," European Radiology, vol. 14, no. 12, pp. 2347-2356, 2004. 


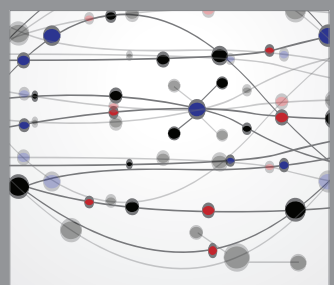

The Scientific World Journal
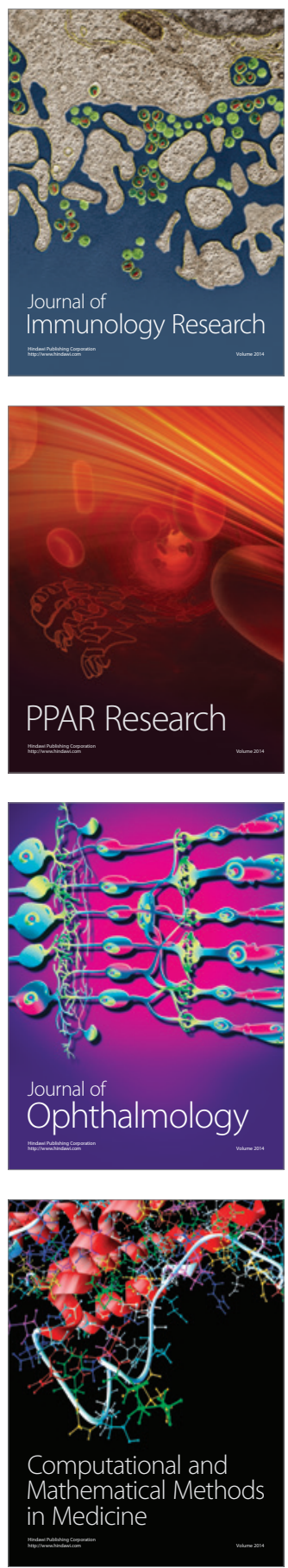

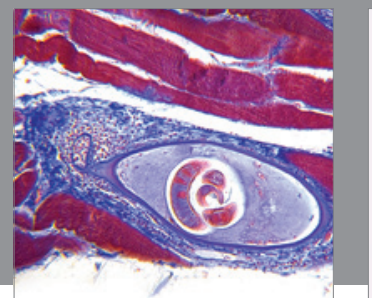

Gastroenterology

Research and Practice
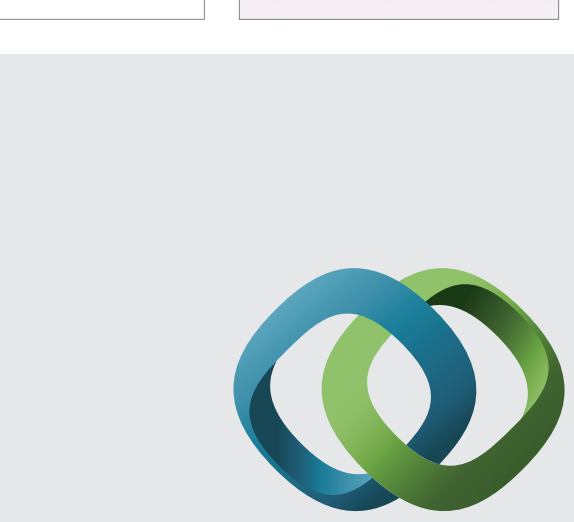

\section{Hindawi}

Submit your manuscripts at

http://www.hindawi.com
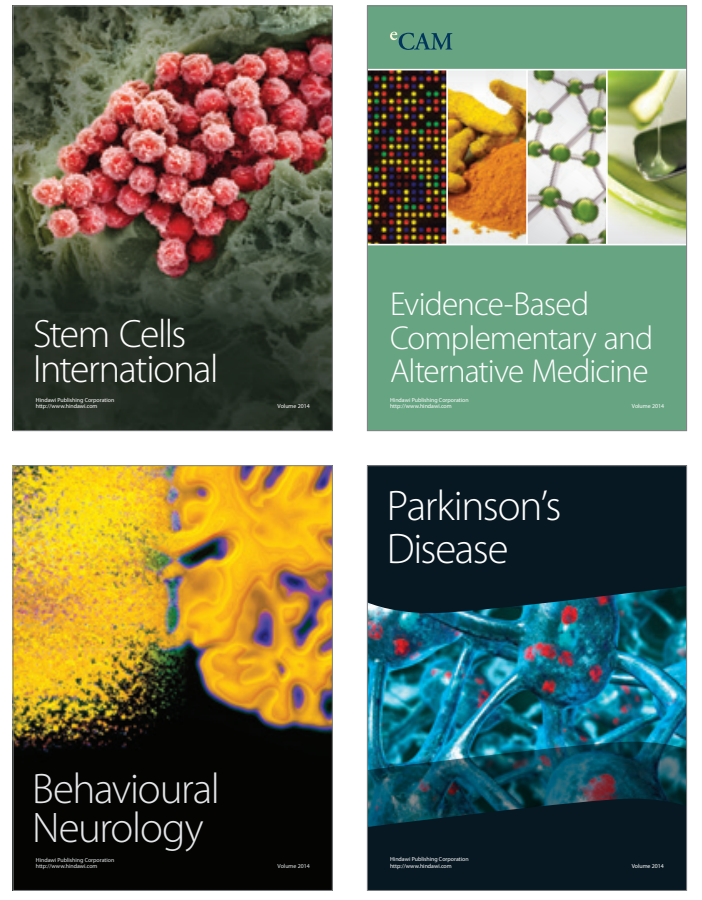
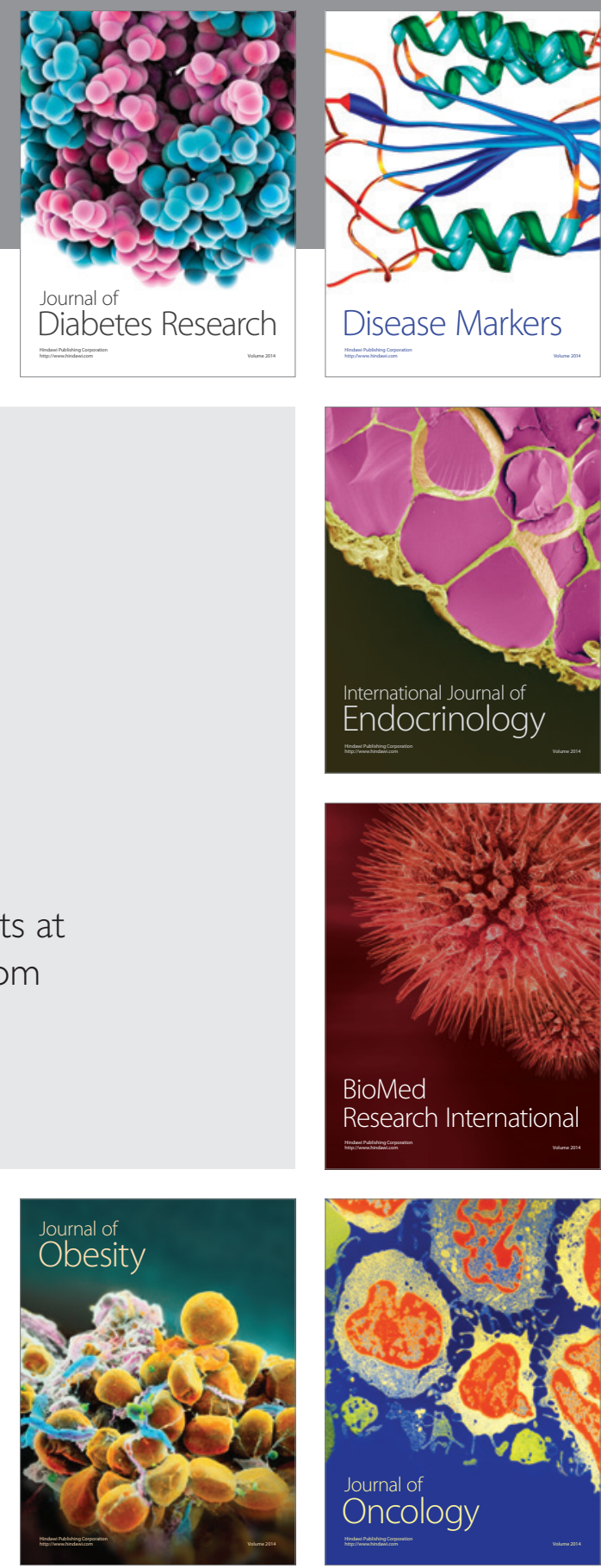

Disease Markers
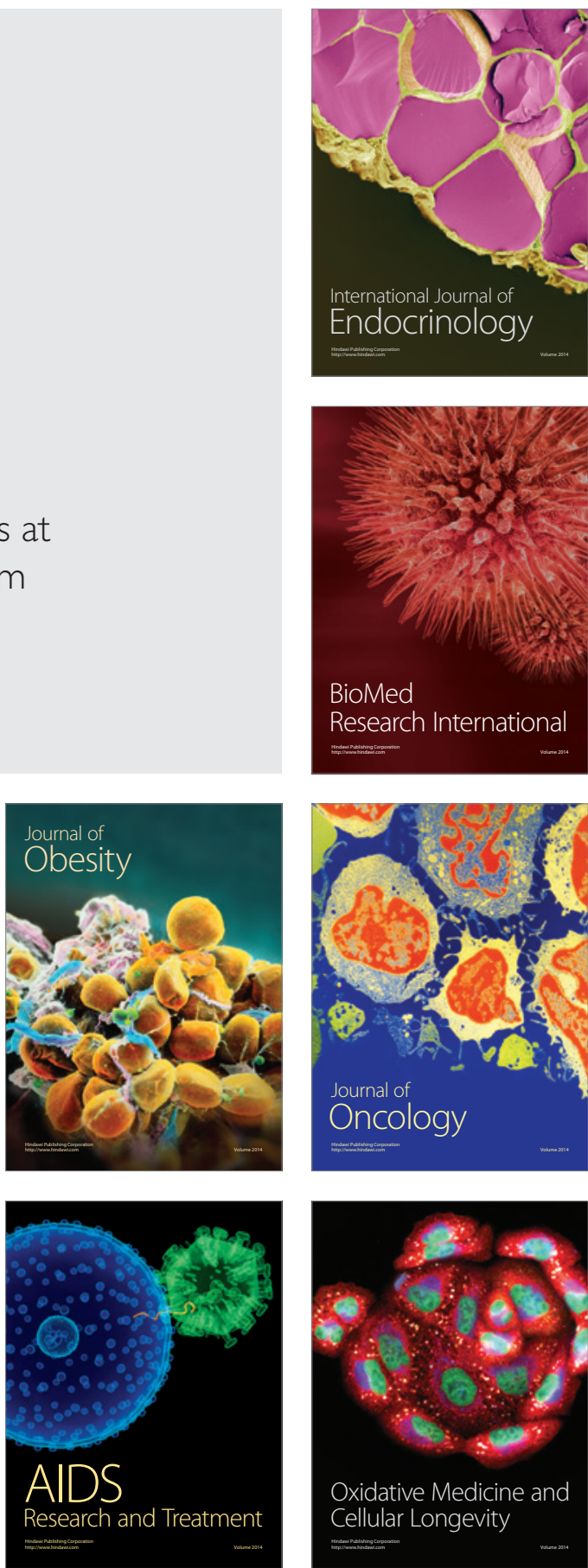